\title{
3. Zur Kinematik \\ des starren Körpers in der Relativtheorie ${ }^{1}$; von Fritz Noether.
}

\$ 1. Die Bornsche Differentialbedingung und die Translationen des starren Körpers.

Die Definition eines starren Körpers in der Newtonschen Mechanik beruht auf dem Grundprinzip, daB die Gleichzeitigkeit zweier Ereignisse eine durch gleichförmige Translation des Bezugssystems unzerstörbare Eigenschaft ist, und betrachtet dementsprechend einen Körper als starr, wenn je zwei seiner Punkte ihre bei gleicher Zeit gemessene Entfernung zeitlich nicht verändern. Diese Forderung ist aber nicht der von Lorentz, Einstein und Minkowski im Anschluß an die Entwicklung der Elektrodynamik begründeten Relativtheorie angepaBt, in der die Invarianz der Naturgesetze gegenüber denjenigen linearen Transformationen der Raumund Zeitkoordinaten postuliert wird, die die Lichtgeschwindigkeit im Vakuum unverändert lassen (Lorentztransformationen).

Deshalb hat Hr. M. Born ${ }^{2}$ ) eine neue Definition für die Bewegung eines als starr zu bezeichnenden Körpers aufzustellen versucht, die die Forderung der Invarianz gegenüber Lorentztransformationen erfüllt. Nach der Relativtheorie hat bekanntlich nur die Gestalt eines (gleichförmig bewegten) Körpers Bedeutung, wie sie von einem relativ zum Körper in Ruhe befindlichen Koordinatensystem aus erscheint, die „Ruhgestalt“" des Körpers, von einem anderen Bezugssystem aus gesehen bängt sie von der relativen Bewegung gegen dieses System

1) Die vorliegende Arbeit ist unabbängig von der von Herglotz über das gleiche Thema (Ann. d. Phys. 31. p. 393. 1910), von deren Druck. legung ich erst während der letzten Ausarbeitung des Manuskriptes erfuhr.

2) M. Born, Ann. d. Phys. 30. p. 1. 1909 u. Physik. Zeitschr. 10. p. 814.1909 . 
ab. Entsprechend muB bei quasistationärer Bewegung die von einem ruhenden System aus erscheinende Gestalt, der augenblicklichen Geschwindigkeit entsprechend, sich verändern. Die Bornsche Definition dehnt nun die Anschauungen der Relativtheorie auf beschleunigte Bewegungen aus, indem sie einen beliebigen Körper als starr bezeichnet, wenn jedes seiner Volumelemente, von einem festen System aus gesehen, immer unabhängig von seiner Beschleunigung den seiner augenblicklichen Geschwindigkeit entsprechenden Deformationszustand zeigt. Präziser gefaBt: Bei der Bewegung des starren Körpers soll die "Ruhgestalt" der infinitesimalen Umgebung eines beliebigen Punktes dauernd unverändert bleiben.

Die Definition ist zunächst der Translation eines Körpers angepaßt, da eben nur für quasistationäre Translationen das Relativprinzip etwas aussagen kann und die Definition eine Erweiterung dieser Bewegungen za beschleunigten Bewegungen fordert. Für den Fall der geradlinigen Translation, wenn also alle Weltlinien parallel einer festen Ebene des $x, y, z, t$ Raumes verlaufen, hat Hr. Born die angeführte Differentialbedingung der Starrheit integriert, und gelangt so zu der Fassung, die als endliche Bedingung der Starrheit für geradlinige Translation zu bezeichnen ist: ${ }^{1}$ )

Die Bewegung eines Punktsystems ist die geradlinige Translation eines starren Körpers, wenn in einem mitgeführten Lorentzkoordinatensystem, in dem ein Punkt des Körpers ruht, zugleich alle seine Punkte sich in Ruhe befinden. Die Ruhgestalt des Körpers, in diesem System gemessen, bleibt dabei dauernd unverändert. Ersichtlich ist in dieser Fassung die Starrheitsdefinition fast wörtlich mit der der alten Kinematik identisch, und wie dort hat die Bewegung die Eigenschaft, daB durch die Vorgabe der Bewegung eines Punktes die des ganzen Körpers mitbestimmt ist, daB also die allgemeine Darstellung nur eine willkürliche Funktion enthält.

Bei Vorgabe beliebiger krummliniger Bewegung eines der Systempunkte kann offenbar diese Formulierung unmittelbar übertragen werden: Eine Bewegung ist die Translation eines starren Körpers, wenn de Lorentztransformation, die einen seiner

1) l. c. p. 24. 
Punkte zur Ruhe transformiert, zugleich alle Punkte des Körpers auf Ruhe transformiert.

Das Weltlinienbild dieser Bewegung ist so $\mathrm{zu}$ beschreiben: Die Weltlinien bilden die Orthogonaltrajektorien ${ }^{3}$ ) eines einfach unendlichen Systems linearer Räume, eben der $x^{\prime}, y^{\prime}, z^{\prime}$ Räume der aufeinanderfolgenden Ruhkoordinatensysteme der Bewegung. Ein solches Bild ist durch die Vorgabe einer der Weltlinien völlig bestimmt, enthält also im allgemeinen Falle räumlicher Bewegung drei willkürliche Funktionen, wie die Translation der Newtonschen Kinematik und analog zu dieser sind die Räume gleicher Weltlinienrichtung eben diese $x^{\prime}, y^{\prime}, z^{\prime}$. Räume der aufeinanderfolgenden Ruhsysteme. Es bleibt aber auch die Ruhgestalt des Körpers, dio in diesem System gemessene Gestalt, unverändert. Denn da die Weltlinien auf diesen Räumen orthogonal stehen, so kann die augenblickliche Bewegung als eine infinitesimale Drehung um die Schnittebene zweier benachbarter Räume aufgefaBt werden, unter Drehung naturlich eine solche lineare Transformation des $x, y, z, t$ Raumes verstanden, die die vierdimensionale Entfernung zweier Punkte, also die Größe:

$$
r_{12}^{2}=\left(x_{1}-x_{2}\right)^{2}+\left(y_{1}-y_{2}\right)^{2}+\left(z_{1}-z_{2}\right)^{2}-c^{2}\left(t_{1}-t_{2}\right)^{2}
$$

unverändert läBt. Bei dieser Drehung wird aber der erste Raum kongruent in den benachbarten übergeführt, die Ruhgestalt bleibt in der Tat invariant. Durch Spezialisierung folgt, da $B$ die Bornsche Bedingung, die die Invarianz der infinitesimalen Rubgestalt fordert, bei den so definierten Translationen erfüllt ist.

Es zeigt sich also, dab die den Differentialbedingungen genügenden Translationen in äußerst einfacher Weise durch endliche Bedingungen definiert werden können. Die Frage nach den allgemeinsten Lösungen der Differentialbedingungen, die Hr. Born noch offen gelassen hat, also nach den Lösungen, die Translationen und Rotationen umfassen, wird zur Ent-

1) Als ,orthogonal“ sind im folgenden immer im Sinne Mink ow sk is (Phys. Zeitschr. 10. p. 104 und Jahresber. d. deutschen Math. Ver. 18.) zwei Vektoren aufzufassen, die die Bedingung:

erfiillen.

$$
x_{1} x_{2}+y_{1} y_{2}+z_{1} x_{2}-c^{2} t_{1} t_{2}=0
$$


scheidung über die Tragweite der differentiellen Formulierung wesentlich beitragen. Im folgenden werde ich nun zeigen, daB die beschriebenen Translationen, abgesehen von dem als singuläre Lösung zu betrachtenden Falle der gleichförmigen Rotation eben diese gesuchte allgemeinste Lösung darstellen. Irgendwelche beschleunigte Rotationen oder Rotationen um beschleunigt bewegte Punkte sind mit der Bornschen Bedingung nicht verträglich.

\$2. Die Eulersche Form der Differentialgleichungen für die Bornsche Bedingung.

Die Bewegung des starren Körpers kann, als Strömung in dem $x, y, z, t$-Raum aufgefa $B t$, entsprechend den Aufgaben der Hydromechanik durch ein Lagrangesches oder ein Eulersches System von Differentialgleichungen beschrieben werden. Hr. Born hat die erstere Form gewählt, gelangt aber dabei $\mathrm{zu}$ einem komplizierten Gleichungssystem. In dem ausgeführten Falle der geradlinigen Translation ist er daher mittels Transformation zur Eulerschen Form übergegangen. Ich werde im folgenden von der Eulerschen Form der Differentialgleichungen ausgehen, also den Geschwindigkeitsvektor als Funktion des Ortes im $x, y, z, t$-Raume suchen.

Die Koordinaten $x, y, z, t$ beziehen sich auf ein irgendwie gewähltes „ruhendes" System und es sei $\tau$ die „Eigenzeit", auf jeder Weltlinie ron einem beliebigen Anfangspunkt an gemessen. Der Geschwindigkeitsvektor sei, in der Bezeichnung von Hrn. Born:

$$
x_{\tau}=\frac{\partial x}{\partial t}, \quad y_{\tau}=\frac{\partial y}{\partial r}, \quad z_{\tau}=\frac{\partial z}{\partial \tau}, \quad t_{\tau}=\frac{\partial t}{\partial \tau},
$$

so daB die Identität besteht:

$$
x_{\tau}^{2}+y_{\tau}^{2}+z_{\tau}^{2}-c^{2} t_{\tau}^{2}=-c^{2} \text {. }
$$

Ferner sei

$$
x_{\tau \tau}=\frac{\partial^{2} x}{\partial \tau^{2}}, \quad y_{\tau \tau}=\begin{aligned}
& \partial^{2} y \\
& \partial \tau^{3}
\end{aligned}, \quad z_{\tau \tau}=\begin{aligned}
& \partial^{2} z \\
& \partial \tau^{2}
\end{aligned}, \quad t_{\tau \tau}=\frac{\partial^{2} t}{\partial \tau^{2}} .
$$

Auf einer beliebig ausgewählten Weltlinie I (vgl. Figur) seien die Koordinaten im ruhenden System:

$$
\mathfrak{x}, \mathfrak{l}), \mathfrak{z}, \mathfrak{t} ;
$$


Zur Kinematik des starren Körpers in der Relativtheorie. 923

in einer beliebig vorgegebenen Richtung $r$ und der Entfernung $d r$ liege der Punkt

$$
x, y, z, t \text {. }
$$

$\mathrm{Zu}$ einer Eigenzeit $\tau_{0}$ der Weltlinie I schneide der Normalraum in $x, y, z, t$ aus der durch $x, y, z, t$ gehenden Weltlinie II den Punkt

$$
x_{1}, y_{1}, z_{1}, t_{1}
$$

mit der von $x, y, z, t \mathrm{ab}$ gerechneten Eigenzeit $d \tau_{1}$ aus, entsprechend schneide zu der auf I gerechneten Eigenzeit $\tau_{0}+d \tau_{0}, z u$ der

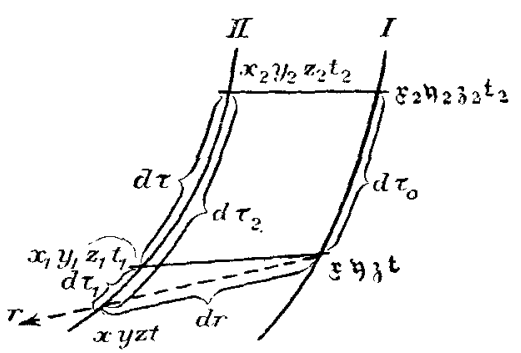

$$
\mathfrak{x}_{2}, \mathfrak{l}_{2}, \mathfrak{z}_{2}, \mathrm{t}_{2}
$$

die Koordinaten auf I seien, der durch" diesen Punkt gelegte Normalraum den Punkt

$$
x_{2}, y_{2}, z_{2}, t_{2}
$$

auf II aus, mit der Eigenzeit $d \boldsymbol{\tau}_{2}$ von $x, y, z, t$ ab gerechnet. Die Differenz der Eigenzeiten auf II sei

Dabei ist $\mathrm{zu}$ setzen:

$$
d \tau=d \tau_{2}-d \tau_{1}
$$

$(2)\left\{\begin{array}{l}x_{1}=x+x_{\tau} d \tau_{1}+\frac{1}{2} x_{\tau \tau} d \tau_{1}{ }^{2} \ldots, \quad x_{2}=\tau+x_{\tau} d \tau_{0}+\frac{1}{2} x_{\tau \tau} d \tau_{0}{ }^{2} \ldots, \\ x_{2}=x+x_{\tau} d \tau_{2}+\frac{1}{2} x_{\tau \tau} d \tau_{2}{ }^{2} \ldots, \\ \text { ferner: } \quad x_{\tau}=x_{\tau}-\frac{\partial x_{\tau}}{\partial r} d r \ldots\end{array}\right.$

und entsprechend für die anderen drei Koordinaten.

Die Eigenzeit $d \tau_{1}$ ist aus der Bedingung bestimmt, dab der Punkt $x_{1}, y_{1}, z_{1}, t_{1}$ in dem Normalraum im Punkt $\mathfrak{z}, \mathfrak{y}, \mathfrak{z} . t$ liegen soll, also dab

$$
\left.\left(x_{1}-x\right) x_{\tau}+\left(y_{1}-\mathfrak{y}\right) \mathfrak{y}_{\tau}+\left(z_{1}-z\right) j_{\tau}-c^{2} \cdot t_{1}-1\right) \mathfrak{t}_{\tau}=0
$$

sei. Hieraus folgt bei Benutzung von (2) und der Identität (1), wenn die Quadrate von $d \tau_{1}$ und $d r$ vernachlässigt werden:

$$
c^{2} d \tau_{1}=(x-d) x_{\tau}+(y-y) y_{\tau}+(z-z) z_{\tau}-c^{2}(t-t) t_{\tau} .
$$

$d \tau_{1}$ ist also von der nämlichen GröBenordnung wie $d r$ (vgl. Gleichung (3a), p. 925). 
Den Wert der Eigenzeit $d \tau_{2}$, der in analoger Weise $z u$ berechnen wäre, werden wir nicht benötigen, es 'genügt zu wissen, daß die Differenz

$$
d \tau-d \tau_{0}=d \tau_{2}-d \tau_{1}-d \tau_{0}
$$

jedenfalls mit $d r$ und mit $d \tau_{0}$ verschwindet und daher von der GröBenordnung $d r . d \tau_{0}$ sein muB.

Die differentielle Starrheitsbedingung ist nun, daß:

$$
\begin{aligned}
& \left(x_{1}-\mathfrak{x}\right)^{2}+\left(y_{1}-\mathfrak{y}\right)^{2}+\left(z_{1}-z\right)^{2}-c^{2}\left(t_{1}-t\right)^{2} \\
= & \left(x_{2}-x_{2}\right)^{2}+\left(y_{2}-\mathfrak{y}_{2}\right)^{2}+\left(z_{2}-z_{2}\right)^{2}-c^{2}\left(t_{2}-t_{2}\right)^{2} .
\end{aligned}
$$

In diese Gleichung sind die Gleichungen (2) einzusetzen, so daB entsteht:

(4a) $\left\{\begin{aligned} & \sum\left(x+x_{\tau} d \tau_{1}+\frac{1}{2} x_{\tau \tau} d \tau_{1}{ }^{2} \ldots-x\right)^{2} . \\ = & \sum\left(x+x_{v} d \tau_{2}+\frac{1}{2} x_{\tau \tau} d \tau_{2}{ }^{2} \ldots-x-\mathfrak{x}_{\tau} d \tau_{0}-\frac{1}{2} x_{\tau \tau} d \tau_{0}{ }^{2} \ldots\right)^{2} .\end{aligned}\right.$

Die Zeichen $\Sigma$ beziehen sich auf die Kombination:

$$
x^{2}+y^{2}+z^{2}-c^{2} t^{2} \text {. }
$$

Unter Beriicksichtigung der Identität (1) ergibt sich aus (4a):

$$
\left\{\begin{array}{l}
2 \sum(x-x) x_{x} d \tau-2 \sum(x-x) x_{\tau} d \tau_{0} \\
\quad+c^{2}\left(d \tau_{1}{ }^{2}-d \tau_{2}{ }^{2}-d \tau_{0}{ }^{2}\right)-2 \sum x_{\tau} x_{\tau} d \tau_{2} d \tau_{0} \\
\quad+\sum(x-x) x_{\tau \tau}\left(d \tau_{2}{ }^{2}-d \tau_{1}{ }^{2}\right)-\sum(x-x) x_{\tau \tau} d \tau_{0}{ }^{2}=0
\end{array}\right.
$$

Es sollen nun mit $r_{1}, r_{2}, r_{3}, r_{4}$ die Richtungskomponenten der Richtung $r$ :

$$
(x-r) / d r, \quad(y-\mathrm{b}) / d r, \quad(z-3) / d r, \quad(t-t) / d r
$$

bezeichnet werden, und die Komponente des Geschwindigkeitsvelktors $x_{\tau}, y_{\tau}, z_{\tau}, t_{t}$ im Punkte $x, y, z, t$ nach dieser Richtung mit:

$$
r_{\tau}=r_{1} x_{\tau}+r_{2} y_{t}+r_{3} z_{\tau}-c^{2} r_{4} t_{\imath}
$$

die des Geschwindigkeitsvektors $\mathfrak{x}_{\tau}, \mathfrak{t}_{r}, \mathfrak{z}_{z}, \mathrm{t}_{z}$ im Punkte $x, \mathfrak{y}, \mathfrak{z} . t$ mit:

$$
\mathfrak{r}_{\tau}=r_{1} \mathfrak{x}_{\tau}+r_{z} \mathfrak{y}_{\tau}+r_{3} z_{\tau}-c^{2} r_{4} \mathfrak{t}_{\tau} .
$$

Entsprechend sei die Komponente des Beschleunigungsvektors nach der festen Richtung $r$ :

$$
\begin{aligned}
& r_{\tau \tau}=d r / d \tau=r_{1} x_{\tau \tau}+r_{2} y_{\tau \tau}+r_{3} z_{\tau \tau}-c^{2} r_{4} \gamma_{\tau \tau}, \\
& r_{\tau \tau}=d r / d \tau=r_{1} x_{\tau \tau}+r_{2} y_{\tau \tau}+r_{3} z_{\tau \tau}-c^{2} r_{4} \ddagger_{\tau \tau},
\end{aligned}
$$


Zur Kinematik des starren Körpers in der Relativtheorie. 925

so daß die Gleichung $(4 \mathrm{~b})$ übergeht in:

$$
\left\{\begin{array}{r}
2\left(r_{\tau} d \tau-r_{\tau} d \tau_{0}\right)+r_{\tau \tau}\left(d \tau_{2}{ }^{2}-d \tau_{1}{ }^{2}\right)-\mathfrak{r}_{\tau \tau} d \tau_{0}{ }^{2} \\
+\frac{e^{2}\left(d \tau_{1}{ }^{2}-d \tau_{2}{ }^{2}-d \tau_{0}{ }^{2}+2 d \tau_{2} d \tau_{0}\right)}{d r}=0 .
\end{array}\right.
$$

Dabei ist noch berücksichtigt, daß nach (1):

$$
x_{\tau} \frac{\partial x_{\tau}}{\partial r}+y_{\tau} \frac{\partial y_{\tau}}{\partial r}+z_{\tau} \frac{\partial z_{\tau}}{\partial r}-c^{2} t_{\tau} \frac{\partial t_{\tau}}{\partial r}=0 .
$$

In der Gleichung (5) kann $d \tau_{2}$ durch $d \tau+d \tau_{1}$ ersetzt werden. Über die GröBenordnung der vorkommenden Differentiale gilt nun, wenn $d r$ und $d \tau_{0}$ als voneinander unabhängige GröBen erster Ordnung betrachtet werden: $d \tau_{1}$ ist nach (3) von der Ordnung $d r$ und zwar wird (3) in der jetzigen Schreibweise:

$$
c^{2} d \tau_{1}=r_{\tau} d r .
$$

Die Differenz $d \tau-d \tau_{0}$ ist, wie schon früher bemerkt, von der Ordnung $d r . d \tau_{0}$. Berücksichtigt man dies und behält in der Gleichung (5) nur die Glieder der Ordnung $d r . d \tau_{0}$ bei, so wird aus (5):

$$
r_{\tau} d \tau-\mathfrak{r}_{\tau} d \tau_{0}+r_{\tau \tau} d \tau d \tau_{1}-c^{2} \frac{d \tau_{1}}{d r}\left(d \tau-d \tau_{0}\right)=0 .
$$

Bei Benutzung von (3a) kann die Gleichung $(6)$ geschrieben werden:

$$
\left(r_{r}-r_{\tau}\right) d \tau+r_{\tau}\left(d \tau-d \tau_{0}\right)+r_{\tau \tau} r_{\tau} d \tau \frac{d r}{e^{2}}-r_{\tau}\left(d \tau-d \tau_{0}\right)=0 \text {, }
$$

also folgt endlich durch Übergang zur Grenze $d r=0, d \tau=0$ :

$$
c^{2} \frac{\partial r_{\tau}}{\partial r}+r_{r} r_{x x}=0 \text {. }
$$

Bei der Ableitung war die Richtung $r$ als eine beliebige in dem vierdimensionalen Raume angenommen. Die Gleichung (7) stellt daher in vektorieller Fassung die Eulersche Form der geforderten Differentialbedingungen dar.

Aus dem Differentialsystem (7) kann sofort eine wesentliche Integraleigenschaft gefolgert werden. Handelt es sich nämlich um ein Gebiet regulärer Bewegung, so ist $r_{z \tau}$, die Beschleunigungskomponente nach der festen Richtung $r$, eine überall endliche analytische Funktion. Dann folgt aber aus (7), daB mit $r_{x}$ auch $\partial r_{\tau} / \partial r$ verschwindet, und weiter kann aus einer Gleichung der Form (7):

$$
\frac{\partial r_{\tau}}{\partial r^{-}}+r_{\tau}^{-} \cdot f=0
$$


wobei $f$ eine endliche, analytische Funktion bedeutet, durch Potenzentwicklung streng gefolgert werden, daB die Funktion $r_{\tau}$ für jeden Wert ron $r$ verschwindet, wenn sie an einer beliebigen Stelle im Endlichen Null ist. Da nun $r_{x}$ die Komponente des Bewegungsvektors nach der Richtung $r$ ist, so folgt der Satz: "Line Gerade, die an einer Stelle auf einer Weltlinie senkrecht steht, muß überall auf den Welttinien senkrecht stehen".

\section{S3. Die Mannigfaltigkeit der Differentialbedingungen.}

Im folgenden soll der Symmetrie halber die Koordinate und das Längenelement:

$$
l=i c t \text {. }
$$

$$
d s=i c d \tau=\sqrt{ } d x^{2}+d y^{2}+d z^{2}+d l^{2}
$$

eingeführt werden, so daß das Gleichungssystem ( 7 ) die Form aunimmt:

$$
\frac{\partial r_{s}}{\partial r}-r_{s} r_{s s}=0
$$

Dabei ist entsprechend der früheren Bezeichnung:

und aus (1) entsteht:

$$
r_{s}=\frac{\partial r}{\partial s}, \quad r_{s s}=\frac{\partial^{2} r}{\partial s^{2}}
$$

$$
x_{s}^{2}+y_{s}^{2}+z_{s}^{2}+l_{s}^{2}=1 .
$$

a) Geradlinige Bewegung.

Im Falle nur zweier Koordinaten $x, l$ ist die Gleichung (8) für jeçe Richtung $r$ erfüllt, wenn sie für eine Richtung erfüllt ist und ist zugleich mit der Inkompressibilitätsbedingung:

identisch.

$$
\frac{\partial x_{s}}{\partial x}+\frac{\partial l_{s}}{\partial l}=0
$$

Denn dann ist wegen $x_{s}{ }^{2}+l_{s}^{2}=1$ :

$$
x_{s} \frac{\partial x_{s}}{\partial x}+l_{s} \frac{\partial l_{s}}{\partial x}=0, \quad x_{s} \frac{\partial x_{k}}{\partial l}+l_{s} \frac{\partial l_{s}}{\partial l}=0
$$

ferner ist identisch:

$$
x_{s s}=x_{s}-\frac{\partial x_{s}}{\partial x}+l \frac{\partial x_{s}}{\partial l}
$$


Zur Kinematik des starren Körpers in der Relativtheorie. 927

also:

d. i.

$$
\begin{aligned}
& \frac{\partial x_{s}}{\partial x}-x_{s} x_{s s}=\dot{\partial x_{s}}-x_{s}\left(\begin{array}{c}
x_{s} x_{s} x_{s}+l_{s} \dot{\partial} x_{s} \\
\partial x^{\prime}
\end{array}\right) \\
& =\left(1-x_{s}{ }^{2}\right)^{\frac{\partial x_{s}}{\partial x}}+l_{s}{ }^{2} \frac{\partial l_{s}}{\partial l},
\end{aligned}
$$

Da nun

$$
\frac{1}{1-x_{s}^{2}}\left(\frac{\partial x_{s}}{\partial x}-x_{s} x_{s s}\right)=\frac{\partial x_{s}}{\partial x}+\frac{\partial l_{s}}{\partial l}
$$

$$
\frac{\partial x_{s}}{\partial x}+\frac{\partial l_{s}}{\partial l}=\operatorname{div} \mathfrak{v}
$$

(wo $\mathfrak{y}$ den Geschwindigkeitsvektor bezeichnet) einen rom Koordinatensystem unabhängigen Wert hat, so folgt entsprechend für eine beliebige Richtung $r$ :

$$
\frac{1}{1-r_{s}^{2}}\left(\frac{\partial r_{s}}{\partial r}-r_{s} r_{s s}\right)=\frac{\partial x_{s}}{\partial x}+\frac{\partial l_{s}}{\partial l} \text {. }
$$

Im Falle der geradlinigen Translation kann also das System (8), durch die eine Inkompressibilitätsbedingung

$$
\frac{\partial x_{s}}{d x}+\frac{\partial l_{s}}{\partial l}=0
$$

mit der Nebenbedingung:

$$
x_{s}^{2}+l_{s}^{2}=1
$$

ersetzt werden $k^{1}{ }^{1}$, dessen allgemeine Lösung eine willkürliche Funktion enthalten mub.

b) Ebene Bewegung.

Es seien $r_{1}, r_{2}, r_{3}$ die Richtungskosinus der willkürlichen Richtung $r$, dann ist

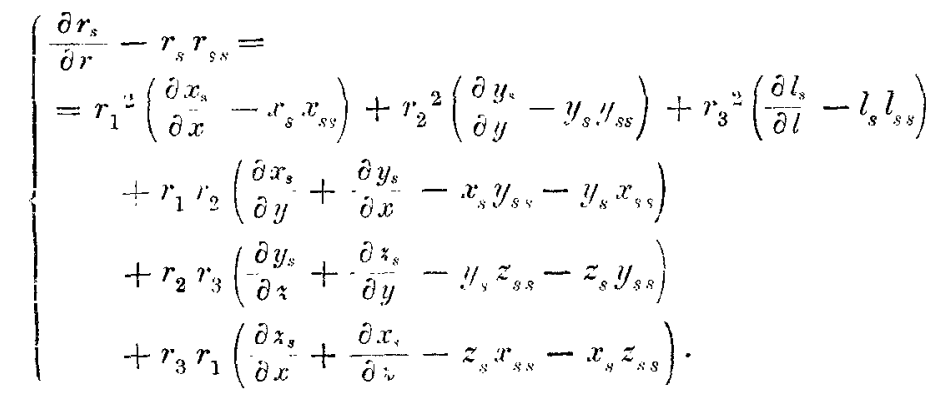

1) Von dieser ist auch Hr. Born bei der Behandlung der geradlinigen Translation ausgegangen, l. e. p. 20. 
Aus der Beziehung:

$$
r_{s s}=x_{s} \frac{\partial r_{s}}{\partial x}+y_{s} \frac{\partial r_{s}}{\partial y}+l_{s}^{\partial r_{s}},
$$

der Gleichung (1a) und den daraus folgenden:

$$
\left\{\begin{array}{l}
x_{s} \frac{\partial x_{s}}{\partial x}+y_{s} \frac{\partial y_{s}}{\partial x}+l_{s} \frac{\partial l_{s}}{\partial s}=0 \\
x_{s} \frac{\partial x_{s}}{\partial y}+y_{s} \partial y_{s}+l_{s} \frac{\partial l_{s}}{\partial y}=0 \\
x_{s} \frac{\partial x_{s}}{\partial x}+y_{s} \frac{\partial y_{s}}{\partial z}+l_{s} \frac{\partial l_{s}}{\partial z}=0
\end{array}\right.
$$

läBt sich nun leicht die Identität folgern:

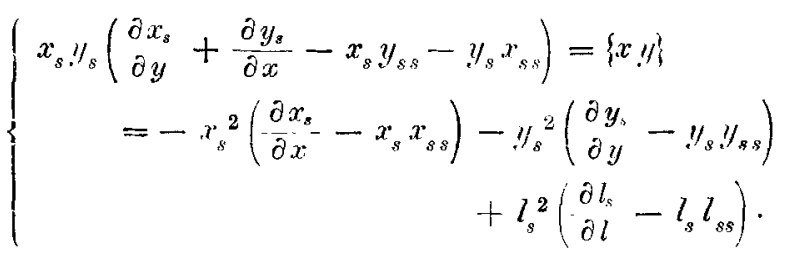

Entsprechende Gleichungen gelten für die aus $\{x y\}$ durch zyklische Vertauschung entstehenden Größen $\{y l\}$ und $\{l x\}$. Dann folgt also aus (9) und (11), daß die Gleichung

$$
\frac{\partial r_{s}}{\partial r}-r_{s} r_{s s}=0
$$

für jede Richtung $r$ besteht, wenn sie für drei Richtungen, etwa $x, y, l$ erfüllt ist.

Diese drei Gleichungen aber sind unabhängig voneinander, denn sie lauten ausgeschrieben, unter Berücksichtigung von (10a):

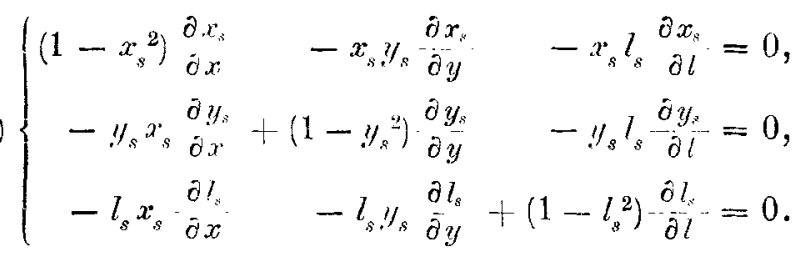

Diese bilden zusammen mit den drei Gleichungen (10) ein System von sechs linearen Gleichungen mit den neun Variabeln

$$
\frac{\partial x_{s}}{\partial x}, \quad \frac{\partial x_{s}}{\partial y} \text { usw. }
$$


Sollte eine Abhängigkeit bestehen, so müBten alle sechsreihigen Determinanten dieses Systems, das sind die der folgenden Matrix, identisch verschwinden:

$\left|\begin{array}{ccccccccc}1-x_{s}{ }^{2} & -x_{s} y_{s} & -x_{s} l_{s} & 0 & 0 & 0 & 0 & 0 & 0 \\ 0 & 0 & 0 & -y_{s} x_{c} & 1-y_{s}{ }^{2} & -y_{s} l_{s} & 0 & 0 & 0 \\ 0 & 0 & 0 & 0 & 0 & 0 & -l_{s} x_{s} & -l_{s} y_{s} & 1-l_{s}{ }^{2} \\ x_{s} & 0 & 0 & y_{s} & 0 & 0 & l_{s} & 0 & 0 \\ 0 & x_{s} & 0 & 0 & y_{s} & 0 & 0 & l_{s} & 0 \\ 0 & 0 & x_{s} & 0 & 0 & y_{s} & 0 & 0 & l_{s}\end{array}\right|$

Man berechnet aber leicht z. B. für die aus der 1., 5., 9., 2, 6., 7. Kolonne gebildete Determinante

$$
\Delta_{i 348)}=x_{s} y_{s} l_{s}\left[x_{s}^{2} y_{s}^{2}+y_{s}{ }^{2} l_{s}^{2}+l_{s}^{2} x_{s}{ }^{2}\right] \neq 0
$$

Also bilden die drei Gleichungen (12) zusammen mit der Relation

$$
x_{s}^{2}+y_{s}^{2}+l_{s}^{2}=1
$$

ein unabhängiges System von Differentialgleichungen. Es ist daher nicht $z u$ erwarten, daB die allgemeine Lösung des Systems, wie in der alten Kinematik, drei willkürliche Funktionen enthält; handelt es sich doch um drei partielle Differentialgleichungen mit drei unabhängigen und nur zwei abhängigen Variabeln, nämlich den Funktionen $x_{s}, y_{s}, l_{s}$, zwischen denen die Beziehung (1 a) besteht. Bei einem solchen überbestimmten System kann über die Mannigfaltigkeit der Lösungen von vornherein nichts ausgesagt werden.

Um auch den Spezialfall der Newton schen Kinematik, also den Fall $c=\infty$, mit einzubegreifen, ist es notwendig, auf das System (7) zurückzugehen. Für diesen Fall geht zunächst die Gleichung (1) über in

$$
t_{\tau}=1 \text {, }
$$

und damit wird die der $t$-Richtung entsprechende Komponente des Systems (7)

$$
c^{2} \frac{\partial c t_{\tau}}{\partial c t}+c t_{\tau} \cdot c t_{\tau \tau}=c^{2}\left(\frac{\partial t_{v}}{\partial t}+t_{\tau} t_{\tau \tau}\right)=0
$$

identisch erfült. Die anderen Komponenten gehen über in

$$
\frac{\partial r_{t}}{\partial r}=0
$$


eine Vektorgleichung, die durch die drei, jetzt unabhängigen Gleichungen (12a) ersetzt wird:

$$
\frac{\partial x_{\tau}}{\partial x}=0 ; \quad \frac{\partial y_{\tau}}{\partial y}=0 ; \quad \frac{\partial x_{\tau}}{\partial y}+\frac{\partial y_{\tau}}{\partial x}=0 .
$$

Man findet diese, indem man genau wie in (9) die Vektorgleichung für eine beliebige Richtung $r=r_{1} x+r_{2} y$ ansetzt. Die letzte Gleichung entspricht hier der aus (11) folgenden $\{x y\}=0$, die aber dort eine Folge der vier unabhängigen Gleichungen (1a) und (12) ist, während hier (1 b) und (12a) vier unabhängige Gleichungen sind.

Da die Abhängigkeitsverhältnisse also andere sind als im allgemeinen Fall, ist kein Widerspruch darin zu erblicken, daß die Mannigfaltigkeit der Lösungen in diesem Spezialfall eine höhere werden kann.

Ganz analoge Verhältnisse bestehen natürlich auch im Falle von vier Dimensionen, also für die räumliche Bewegung, doch beschränken wir uns der Einfachheit halber vorläufig auf die ebenen Bewegungen.

\section{\$ 4. Fbene Rotation um einen ruhenden Punkt.}

Für die ebene Kotation um einen ruhenden, bzw. gleichförmig bewegten Punkt $O$ ist dessen Weltlinie $l$-Achse eines ruhenden Koordinatensystems. Die durch diese Achse senkrecht zu ihr gelegten Strahlen müssen nach dem Satze auf p. 926 überall auf den Weltlinien senkrecht stehen. Das Verschwinden der Geschwindigkeitskomponente längs eines solchen Strables,

$$
\varrho_{s}=\frac{x}{\varphi} x_{s}+y_{0}^{\prime} y_{s}
$$

wobei $\varrho^{2}=x^{2}+y^{2}$ gesetzt ist, bedeutet aber, daß alle Weltlinien auf Kreiszylindern um die $l$-Achse verlaufen müssen, oder daB vom Ruhsystem des Punktes $O$ aus gesehen alle Punkte sich auf Kreisen um $O$ bewegen.

In einer kürzlich erschienenen Note ${ }^{1}$, in der Hr. P. Ehrenfest auf Widersprïche der Bornschen Differentialbedingung hinweist, scheint mir diese Eigenschaft der Rotationsbewegung

1) P. Ehrenfest, Physik. Zeitschr. 10. p. 918, 1909. 
ohne Beweis angenommen zu sein. Für die Frage der Irrtegrabilität der Differentialgleichungen folgt aus dem Ehrenfestschen Einwand schon, daß der Übergang von der Ruhe zur gleichförmigen Rotation um einen ruhenden Punkt nicht durch eine beim Umlaufen der Weltlinie des ruhenden Punktes eindeutige Lösung dargestellt werden kann.

Inwieweit Drehungen um einen ruhenden Punkt möglich sind, ergibt sich nun einfach durch die folgende geometrische Betrachtung: An einer beliebigen Stelle $P$ eines vorgegebenen Kreiszylinders um die $l$-Achse kann die Neigung des Weltlinienelementes, d. h. die Rotationsgeschwindigkeit des Punktes $P$, beliebig vorgegeben werden. Die Normalebene dieses Elementes schneide die Ellipse $E_{p}$ aus dem Zylinder aus. Unter $Q$ einen beliebigen Punkt dieser Ellipse verstanden, mub die Verbindungsgerade $P Q$ überall auf den Weltlinien senkrecht stehen, da im Punkt $P$ die Weltlinie auf $P Q$ senkrecht steht. Daher muB das Weltlinienelement in $Q$ die zwei Bedingungen erfüllen.

1. Es $m u B$ in der Zylindertangentialebene in $Q$ liegen.

2. Es muB in der Normalebene zu $P Q$ im Punkte $Q$ liegen.

Durch diese beiden Bedingungen ist seine Richtung völlig bestimmt als Schnitt der Zylindertangentialebene mit der Normalebene zu $P Q$. Das ursprünglich in $P$ angenommene Element genügt offenbar den nämlichen beiden Bedingungen, bei Vertauschung von $Q$ mit $P$. Aus Symmetriegründen (bezüglich der Richtungen ist alles symmetrisch zur Halbierungsebene des Außenwinkels der Zylindertangentialebenen in $P$ und $Q$, zu der die Verbindungsgerade $P Q$ parallel liegt) ist nun klar, daB die Neigung des so bestimmten Bahnelementes in $Q$ die nämliche wird, wie die in $P$, und zwar können beide Elemente durch Drehung und Verschiebung des Zylinders in sich ineinander übergeführt werden. Entsprechend erhält man längs der ganzen Ellipse $E_{P}$ Bahnelemente von gleicher Neigung wie in $P$. Die Normalebene $z \mathfrak{u}$ dem so bestimmten Bahnelement in $Q$ geht durch den Punkt $P$, weil die Gerade $P Q$ senkrecht auf dem Bahnelement in $Q$ steht, schneidet aber im übrigen eine neue Ellipse $E_{Q}$ aus dem Zylinder aus, ausgenommen den Fall, daB das Element in $P$ parallel der Zylinderachse angenommen war, also der Punkt $P$ ruhte. Durch 
Fortsefzung der Konstruktion erhält man längs der Ellipse $E_{Q}$ überall Elemente gleicher Neigung, und wenn man den Punkt $Q$ die Ellipse $E_{P}$ durchlaufen läBt, eine Zone des Zylinders, endlich von dieser weitergebend, den ganzen Zylinder mit Elementen gleicher Neigung überdeckt. Dies ist aber das Weltlinienbild der gleichförmigen Drehung um den ruhenden Punkt. Also kann nur die gleichförmige Drehung eine den Differentialbedingungen genügende Bewegung sein, wenn ein Punkt des Körpers in Ruhe angenommen wird. Das Weltlinienbild ist dann das der Bewegungskurven einer dreidimensionalen, unendlich kleinen Schraubung des $x, y, l$-Raumes, bei dem in der Tat bekanntlich die Bedingung erfüllt ist, dab eine Gerade, die an einer Stelle auf den Bewegungskurven senkrecht steht, dann auch überall auf diesen senkrecht steht.

Die folgende analytische Fassung des Beweises kann leicht (vgl. \$ 5) für die Drehung um einen beliebig bewegten Punkt erweitert werden.

Es sei die Weltlinie des ruhenden Punktes

$$
x=y=0 \text {, }
$$

und für einen beliebigen Punkt sei gesetzt:

$$
x=\varrho \cos \varphi ; \quad y=\varrho \sin \varphi ; \quad l=l .
$$

Nach p. 930 ist $\varrho_{s}=0$, d. h. der Radius $\rho$ längs einer Weltlinie konstant, also ist

$$
x_{s}=-\ell \sin \varphi \cdot \varphi_{s} ; \quad y_{s}=\varrho \cos \varphi \cdot \varphi_{s} .
$$

Wir haben (13) und (13a) in die Gleichungen (12) einzusetzen. Um zunächst die Abhängigkeit des Weltlinienverlaufes von $\mathscr{f}$ und $l$ auf einem Zylinder $\varrho=$ const. zu erhalten, ist es, wie bei der geradlinigen Translation zweckmäBig, eine Kombination der Gleichungen (12), die Inkompressibilitätsgleichung, zu verwenden. Aus den drei unabhängigen Gleichungen (12):

$$
\left\{\begin{array}{l}
\frac{\partial x_{s}}{\partial x}-x_{s} x_{s s}=0, \\
\frac{\partial y_{s}}{\partial y}-y_{s} y_{s s}=0, \\
\frac{\partial l_{s}}{\partial l}-l_{s} l_{s s}=0,
\end{array}\right.
$$


Zur Kinematik des starren Körpers in der Relativtheorie. 933

folgt diese durch Addition unter Berücksichtigung der Identität (1 a) zu:

$$
\frac{\partial x_{s}}{\partial x}+\frac{\partial y_{s}}{\partial y}+\frac{\partial l_{s}}{\partial l}=0 .
$$

Durch Einsetzen der Werte von $x, x_{s}, y, y_{s}$ aus (13) und (13a) in (14) geht diese Gleichung über in

$$
\frac{\partial \varphi_{s}}{\partial \varphi_{i}}+\frac{\partial l_{s}}{\partial l}=0 \text {. }
$$

Unter Berücksichtigung der Beziehung (1 a) endlich, die hier die Gestalt annimmt:

wird daraus:

$$
\rho^{2} \varphi_{s}^{2}+l_{s}^{2}=1
$$

$$
l_{s} \frac{\partial \psi_{s}}{\partial \psi_{p}}-\varphi^{2} \psi_{s} \frac{\partial \psi_{s}}{\partial l}=0 \text {. }
$$

Die geometrische Bedeutung der Gleichung (14) ist nun, daB die Kurven konstanter Neigung, für die also $\varphi_{s}$ einen konstanten Wert hat, auf der Zylinderfläche vom Radius $\varrho$ senkrecht $\mathrm{zu}$ den Weltlinien verlaufen, $\mathrm{da} B$ also die Weltlinien die Orthogonaltrajektorien eines Systems von Isogonalkurven zu den Zylindergeraden sind. Durch Abwickelung der Zylinder ginge dieses Weltlinienbild in das der geradlinigen Translation, p. 920 , über.

Die Abhängigkeit der Neigung $\varrho \varphi_{s}$ vom Radius $\varrho$ ergibt sich offenbar zweckmäBig aus einer anderen Kombination der Gleichungen (12), nämlich der aus (11) und (12) folgenden Beziehung $\{x y\}=0$ (vgl. p. 928); d. h.

$$
\frac{\partial x_{\mathrm{s}}}{\partial y}+\frac{\partial y_{s}}{\partial x}-x_{s} y_{s s}-y_{s} x_{s s}=0,
$$

indem man in dieser Gleichung die Richtungen $x$ und $y$ als die Tangente in der $x y$-Ebene und die Normale der ZylinderHäche in dem gerade betrachteten Punkte wählt, $d . h$. dessen Azimut als den in den Gleichungen (13) willkürlich gelassenen Anfangspunkt $\varphi=0$ wählt. Da dann $\left(y_{s}\right)_{\varphi=0}$ und $\left(\partial y_{s} / \partial l\right)_{\varphi=0}$ verschwindet, vereinfacht $\operatorname{sich}(11 \mathrm{a})$ nach (13) zu:

$$
\frac{\partial \rho \varphi_{s}}{\partial \varphi}+\frac{1}{\rho}\left(\frac{\partial y_{b}}{\partial \varphi}\right)_{4=0}-\varphi \varphi_{s}^{2}\left(\frac{\partial y_{s}}{\partial \varphi}\right)_{f=1}=0 .
$$

Hier ist

$$
\left(\frac{\partial}{\partial \varphi} y_{s}\right)_{r=0}=-\varphi \varphi_{s}
$$


also wird die Gleichung 15 :i:

$$
\frac{\bar{o} q_{s}}{\partial \varphi}+\varphi q_{s}^{3}=0
$$

Durch Integration ergibt sich hieraus:

und also

$$
f_{s}=-\frac{1}{\sqrt{b^{2}+C}}
$$

$$
I_{s}=\frac{C}{V_{q^{2}}+C^{\prime \prime}},
$$

wobei $C=C(\varphi, l$, als von $\varrho$ unabhängig zu bestimmen ist. Die Bedeutung der Gleichung (16) ist, daß sich die Neigung $\varphi \varphi_{s}$ längs des Strahles $\varrho$ wie bei gleicher Winkelgeschwindigkeit $\partial \varphi / \partial l$ der Punkte dieses Strahles verändern muB.

Setat man die Gleichung (16) in (14a) ein, so ergibt sich für $C(p, l)$ die Differentialgleichung:

$$
C \frac{\partial C}{\partial \varphi}-\rho^{2} \frac{\partial C}{\partial l}=0
$$

aus der sich ein von " unabhängiger Wert von $C$ offenbar nur dann ergibt, wenn

$$
\frac{\partial C}{\partial \varphi}=\frac{\partial C}{\partial l}=0
$$

also $C=$ const. und somit $\varphi_{s}$ und $l_{s}$ auf der ganzen Zylinderfläche konstant sind.

Also ist die gleichförmige Rotation die einzige mögliche Lösung der Gleichungen (14a) und (15).

Das Resultat würde natürlich hinfällig werden im Falle $c=\infty$, für den aus dem (12) entsprechenden System $\partial r_{r} / \partial r=0$ wegen $t_{r}=1$ die Koordinate $t$ völlig herausfällt und also durch diese Gleichungen keine Verknüpfung der Bewegungszustände $\mathrm{zu}$ verschiedenen Zeiten gegeben ist.

\$ 5. Ebene Rotation um einen beschleunigt bewegten Punkt.

Es seien die Koordinaten einer beliebig vorgegebenen Weltlinie:

$$
\xi . \lambda,
$$

und ihr Längenelement

$$
d r=\gamma^{2} \xi^{2}+d \eta^{2}+d \lambda^{2} .
$$


Genau wie bei der Bewegung um einen ruhenden Punkt legen wir nun zu dieser Kurve alle Normalebenen und ziehen in diesen Ebenen die Strahlen durch den Punkt $\xi, \eta, \lambda$.

Da diese Strahlen auf der Weltlinie $\xi, \eta, \lambda$ senkrecht stehen, so muß überall die in ihrer Richtung liegende Geschwindigkeitskomponente verschwinden. Daher müssen wieder alle Weltlinien auf "Zylinderflächen" um die gegebene Kurve verlaufen, Flächen, die dadurch charakterisiert sind, dab sie aus jeder der Normalebenen einen Kreis von gleichem Radius ausschneiden. Wir wählen nun zur Festlegung eines beliebigem Punktes

1. den Parameter $\sigma$ der Normalebene, in dem der Punkt liegt, wobei $\sigma$ von einem beliebigem Punkt $\xi_{0}, \eta_{0}, \lambda_{0}$ ab zu zählen ist.

2. Polarkoordinaten $\varphi, \varphi$ in dieser Normalebene, mit dem Durchschnitt der Normalebene mit der Kurre $\xi, n, \lambda$ als Pol, wobei $\varphi$ von der Schmiegungsebene der Kurve $\xi, \eta, \lambda$ ab gezählt werden soll. Es sei ferner $R$ der Krümmungsradius, $T$ der Torsionsradius dieser Kurve.

Die Richtung der Weltlinien auf einer solchen Zylinderfläche ist dann durch die Komponenten

$$
\frac{\partial \varphi}{\partial s}=\varphi_{s} ; \quad \frac{\partial \sigma}{\partial s}=\sigma_{s}
$$

charakterisiert. Die linearen Bewegungskomponenten sind senkrecht zur erwähnten Normalebene, also parallel der gegebenen Kurve $\xi, \eta, \lambda$ :

$$
u=\left(1+\frac{o}{R} \cos \varphi\right) \sigma_{s},
$$

in der Normalebene und zwar senkrecht auf dem Strahl o:

$$
v=\varrho\left(\varphi_{s}+\frac{\sigma_{s}}{T}\right)
$$

in der Normalebene und in der Richtung des Strables 0 :

$$
\theta_{s}=0 \text {. }
$$

Das Weltlinienbild auf der Zylinderfiäche wird nun wieder am einfachsten wie beim ruhenden Punkt durch die aus den Gleichungen (12) durch Addition gebildete Inkompressibilitätsgleichung:

$$
\frac{\partial x_{s}}{\dot{\partial} x}+\frac{\dot{\partial} y_{s}}{\partial y}+\frac{\partial l_{s}}{\partial l}=0
$$


beschrieben, indem man sie in die krummlinigen Koordinaten $\sigma, \varrho, \varphi$ umschreibt. Für die Durchführung der Umrechnung wählt man natürlich zweckmäBig für die Richtungen $x, y, l$ das durch die Richtungen $u, v, \varrho_{s}$ in dem gerade betrachteten Punkt bestimmte geradlinige Achsenkreuz, das das krummlinige Koordinatensystem $\sigma, \varrho, \varphi$ tangiert. Die so entstehende Gleichung lautet $\left.{ }^{1}\right):$

$$
\begin{aligned}
\frac{\partial}{\partial \varphi}\left(\varphi_{s}+\frac{\sigma_{s}}{T}\right) & -\frac{R}{T(R+\varphi \cos \varphi)} \frac{\partial}{\partial \varphi}\left[\left(1+\frac{\varrho}{R} \cos \varphi\right) \sigma_{s}\right] \\
& +-\frac{R}{R+\varphi \cos \varphi} \frac{\partial}{\partial \sigma}\left[\left(1+\frac{\varrho}{R} \cos \varphi\right) \sigma_{s}\right] \\
& -\varphi\left(\varphi_{s}+\frac{\sigma_{s}}{T}\right) \frac{\sin \varphi}{R+\varrho \cos \varphi}=0 .
\end{aligned}
$$

Mittels der Gleichung (1a), die hier die Form annimmt:

$$
u^{2}+v^{2}=\left(1+\frac{g}{L} \cos \varphi\right)^{2} \sigma_{s}^{2}+\varphi^{2}\left(\varphi_{s}+\frac{\sigma_{s}}{T}\right)^{2}=1
$$

wird daraus:

$$
\left\{\begin{aligned}
{\left[\left(1+\frac{\rho}{R} \cos \varphi\right)^{2} \sigma_{s}\right.} & \left.+\frac{\underline{Q}^{2}}{T}\left(\varphi_{s}+\frac{\sigma_{s}}{T}\right)\right] \frac{\partial}{\partial \varphi}\left(\varphi_{s}+\frac{\sigma_{s}}{T}\right) \\
& -\varrho^{2}\left(\varphi_{s}+\frac{\sigma_{s}}{T}\right) \frac{\partial}{\partial \sigma}\left(\varphi_{s}+\frac{\sigma_{s}}{T}\right) \\
& -\left(\frac{\varrho}{R} \cos \varphi\right) \frac{\rho}{R} \sin \varphi\left(\varphi_{s}+\frac{\sigma_{s}}{T}\right) \sigma_{s}=0 .
\end{aligned}\right.
$$

Die Gleichung (17) kann, in Übereinstimmung mit einer der geometrischen Überlegung auf p. 931 analogen Betrachtung so gedeutet werden:

Die Weltlinien bilden auf der Zylinderfläche die Orthogonaltrajektorien eines einfach unendlichen Systems geodätischer Linien.

1) Es ist dann nämlich an der betrachteten Stelle $(P)$ :

$$
\begin{gathered}
\frac{\partial}{\partial x}=\frac{1}{\varrho} \frac{\partial}{\partial \varphi} ; \quad \frac{\partial}{\partial y}=-\frac{\partial}{\partial \varrho} ; \quad \frac{\partial}{\partial l}=\frac{I}{R+\rho \cos \varphi}\left(\frac{\partial}{\partial \sigma}+\frac{1}{T} \frac{\partial}{\partial \varphi}\right), \\
\left(x_{s}\right)_{P}=v ; \quad\left(y_{s}\right)_{P}=\rho_{s}=0 ; \quad\left(I_{s}\right)_{P}=u .
\end{gathered}
$$

Da $y_{s}=0$, so ist trotz der Krümmung des Koordinatensystems $\partial x_{s} / \partial x$ aus diesen Formeln unmittelbar zu bilden, in $\partial l_{s} / \partial l$ dagegen tritt ein Zusatzglied nach Art der Zentrifugalglieder hiszu:

$$
\frac{\partial l_{s}}{\partial l}=\frac{\partial\left(l_{s}\right)_{P}}{\partial l}-x_{s} \frac{\sin \varphi}{R+\rho \cos \phi} .
$$


Um nun noch die Abhängigkeit des Weltlinienverlaufes vom Zylinderradius $\rho \mathrm{zu}$ erhalten, ist wieder (wie p. 933) die Gleichung (11 a) geeignet:

$$
\frac{\partial x_{s}}{\partial y}+\frac{\partial y_{s}}{\partial x}-x_{s} y_{s s}-y_{s} x_{s, s}=0
$$

indem wir die Richtung $x$ in die Tangentenrichtung $v$, die Richtung $y$ in die Strahlrichtung $\varrho$ legen. Es ist dann also $x_{s}=v$ (p. 935); ferner an der betrachteten Stelle $y_{s}=0$ und $\partial y_{s} / \partial y=0$, dagegen wegen der Krümmung des Koordinatensystems:

$$
\begin{aligned}
\frac{\partial y_{s}}{\partial x}=\frac{1}{\varphi} \frac{\partial y_{s}}{\partial \varphi} & =-\varphi\left(\psi_{s}+\frac{\sigma_{s}}{T}\right) \\
\frac{\partial y_{s}}{\partial \theta} & =-(1+\stackrel{\varrho}{R} \cos \varphi) \frac{\cos \varphi}{R} \sigma_{s}-\frac{\varrho}{P}\left(\psi_{s}+\frac{\sigma_{s}}{T}\right) .
\end{aligned}
$$

Durch Einsetzen dieser Werte in (11a), unter Beachtung, daB

$$
y_{s s}=\sigma_{s} \frac{\partial y_{s}}{\partial \sigma}+\varphi_{s} \frac{\partial y_{s}}{\partial \varphi}
$$

resultiert die $z u(15)$ analoge Gleichung:

$$
\frac{\partial\left(p_{s}+\frac{\sigma_{s}}{T}\right)}{\partial \varphi}+R \cdot \frac{\left(\varphi_{s}+\frac{\sigma_{s}}{T}\right)^{3}}{R+\rho \cos \varphi}-\frac{\left(\varphi_{s}+\frac{\sigma_{s}}{T}\right) \cos \varphi}{R+\varphi \cos \varphi}=0 .
$$

Die Gleichung (18) hat das zu (16) analoge Integral:

und also:

$$
\varphi_{s}+\sigma_{s}^{\sigma_{s}}=\frac{1}{\sqrt{\varphi^{2}+\left(1+\frac{\varrho}{R} \cos \varphi\right)^{2} C^{2}},}
$$

$$
\sigma_{s}=\frac{\frac{C}{\sqrt{g^{2}+\left(1+\frac{\rho}{R} \cos \varphi\right)^{2} C^{2}}}}{}
$$

das auch die nämliche geometrische Bedeutung hat, daB längs eines Strahles $\varrho$ die Winkelgeschwindigkeit $\partial \gamma / \partial \sigma+1 / T$ konstant ist. $C$ muB eine von $\varrho$ unabhängige Funktion $C(\sigma, \varphi)$ sein. Für sie ergibt sich aber durch Einsetzen von (19) in (17) die Gleichung 


$$
\left\{\begin{aligned}
{\left[\left(1+\frac{Q}{L} \cos \varphi\right)^{2}\right.} & \left.-\frac{\varrho^{2}}{T}\right]\left(1+\frac{\rho}{R} \cos \varphi\right) C^{2} \frac{\partial C}{\partial \varphi} \\
& -\varrho^{2}\left(1+\frac{\varrho}{R} \cos \varphi\right) C \frac{\partial C}{\partial \sigma} \\
& +\frac{\sigma^{3}}{R} \sin \varphi\left(1-\frac{C^{2}}{T}\right) C+\varrho^{3} \cos \varphi C \frac{\partial \frac{1}{R}}{\partial \sigma}=0 .
\end{aligned}\right.
$$

Aus (20) folgt $C$ als von o unabhängig nur durch die folgenden Annahmen:

1. $C=0$ :

Dann wird $\sigma_{s}=0$, der Fall entspräche daher der unendlich raschen Rotation und ist auszuschlieBen.

2. $C=\infty$ :

Jetzt wird $\varphi_{s}+\sigma_{s} / T=0$, also $v=0$ (p. 935), und offenbar sind für diese Annahme die Gleichungen (17) und (18) in der Tat erfüllt. Die Weltlinien verlaufen alle parallel der ursprünglich vorgegebenen, $(\xi, \eta, \lambda)$, es handelt sich also um den auf p. 921 beschriebenen Fall der Translation, in dem die Weltlinien die Orthogonaltrajektorien eines Ebenensystems sind.

3. Wenn

$$
\left(1-\frac{C^{2}}{T^{\prime}}\right) \sin \varphi+R \frac{\partial \frac{1}{R}}{\partial \sigma} \cos \varphi=0,
$$

so ist die Lösung

$$
\frac{\partial C}{\partial \psi}=\frac{\partial C}{\partial \sigma}=0
$$

möglich. Aus (21) ergibt sich aber ein von $\sigma$ und $\varphi$ unabhängiger Wert für $C$ nur, wenn

und

$$
\frac{\partial \frac{1}{R}}{\partial \sigma}=0
$$

$$
C^{2}=T=\text { const. }
$$

wenn also die vorgegebene Kurve eine Raumkurve konstanter Torsion und Krümmung, d. h. eine Schraubenlinie konstanter Steighöhe ist. Die Winkelgeschwindigkeit ist dabei durch die Torsion eindeutig bestimmt. In dem Fall ist aber eben die vorgegebene Kurve eine der Weltlinien bei der gleichförmigen Rotation um einen ruhenden Punkt. 
E's folgt also, daß außer den Translationen und der gleichförmigen Rotation um einen ruhenden Punkt überhaupt keine ebenen Bewegungen bestehen, die den Differentialbedingungen genügen können.

\section{§ 6. Räumliche Bewegung.}

Für den Fall von vier Dimensionen $x, y, z, l$ hätte zwar die Durchführung des analogen Beweises keine sachlichen Schwierigkeiten, würde aber zu rechnerischen Weitläufigkeiten führen. Wir beschränken uns daher auf die folgende geometrische Skizzierung des Beweises.

Bei der gleichförmigen Rotation um einen gleichförmig bewegten Punkt bilden die Weltlinien die Bewegungskurven einer unendlich kleinen Schraubung des $x, y, z, l$-Raumes, die Richtungen der Weltlinien können daher in der folgenden Form dargestellt werden, wobei $u, v, w, k$ den Geschwindigkeitsvektor in einem willkürlich gewählten Punkt $O$, dem Anfangspunkt des Koordinatensystems, bezeichnet:

$$
\left\{\begin{array}{l}
\mu x_{\tau}=u+\quad f_{12} y+f_{13} z+f_{14} l, \\
\mu y_{\tau}=v+f_{21} x+f_{23} z+f_{24} l, \\
\mu z_{\tau}=v+f_{31} x+f_{32} y+f_{34} l, \\
\mu l_{\tau}=k+f_{41} x+f_{42} y+f_{43} z
\end{array}\right.
$$

die $f_{i k}$ sind Konstanten, die Komponenten des Sechservektors der Drehung, die die Bedingung

erfüllen.

$$
f_{i k}=-f_{k i}
$$

Die Schraubung ist nun etwa durch die folgenden Bestimmungsstücke festgelegt: Die Bahnrichtung in $O$ soll als $l$-Achse angenommen, also

$$
u=v=w=0
$$

gesetzt werden. Ferner soll die Beschleunigungsrichtung in diesem Punkt bekannt sein, also in dem $O$ benachbarten Punkt $O^{\prime}$ mit den Koordinaten $0,0,0, d l$ soll sein:

$$
\begin{aligned}
& \mu x_{x}=f_{14} d l, \\
& \mu y_{x}=f_{24} d l, \\
& \mu z_{x}=f_{34} d l, \\
& \mu l_{x}=k .
\end{aligned}
$$

Daraus sind zunächst $f_{14}, f_{24}, f_{34}$ bestimmt. 
Ferner sei in einem Punkt $P$ der Achse $x=y=l=0$, für den $z=z_{1}$ sei, die Richtung vorgegeben zu

$$
\begin{aligned}
& \mu x_{\tau}=f_{13} z_{1}, \\
& \mu y_{\tau}=f_{23} z_{1}, \\
& \mu z_{x}=0, \\
& \mu l_{\tau}=k+f_{43}^{\prime} z_{1},
\end{aligned}
$$

so daB auch $f_{13}$ und $f_{83}$ bestimmt werden. Es bleibt nun für die Bewegungsrichtung in einem beliebigen Punkt $\ell$ nur mehr der eine, durch $f_{12}$ gekennzeichnete Freiheitsgrad.

Wir suchen nun eine Minimalzahl von Bestimmungsstücken für das durch die Bornsche Bedingung geforderte Weltlinienbild, die auch durch die bei der vorher betrachteten Bewegungsschraube offenbar erfüllte endliche Forderung ersetzt werden kann: Eine Gerade steht überall auf den Weltlinien senkrecht, wenn sie auf einer von ihnen senkrecht steht.

Im Punkt $O$ kann wieder die Weltinienrichtung als l-Achse und die Beschleunigung, d. h. die Richtung im Punkte $O^{\prime}$ mit den Koordinaten $0,0,0$, $d l$ willkürlich gewählt werden. Für einen beliebigen Punkt $P$ des Raumes $l=0$ hat dann die Weltlinienrichtung zwei Freiheitsgrade, da sie nur auf der Verbindung $P O$ senkrecht stehen muB. Wähit man die Richtung $P O$ zur Achse $x=y=l=0$, so sind diese Freiheitsgrade mit denen im Punkt $P$ bei der vorher besprochenen Bewegungsschraube identisch. Für einen weiteren Punkt $Q$, der im Normalraum des Elementes in $P$ und im Raum $l=0$ liegt, bleibt nur noch ein Freiheitsgrad, denn sein Bahnelement $\mathrm{muB}$ die zwei Bedingungen erfüllen, es muB auf $Q P$ und auf QO senkrecht stehen. Dieser Freiheitsgrad mub aber der nämliche sein, wie im Fall der Bewegungsschraube der durch $f_{12}$ gekennzeichnete in $Q$, eben da die geometrischen Bedingungen, die unserer jetzigen Konstruktion zugrunde liegen, dort auch erfüllt sind.

Von den Elementen in $O, O^{\prime}, P, Q$ ausgehend, gelangt man nun sukzessive zur Bestimmung der Elemente im ganzen Raum $x, y, z, l$. Man hat zunächst die Normalräume in $P, Q$ und $O^{\prime}$ zum Schnitt zu bringen und erhält für jeden Punkt $R$ der Schnittgeraden das Weltlinienelement als die gemeinsame 
senkrechte Richtung auf $R P, R Q$ und $R Q^{\prime}$. Von diesem Elemente in $R$, in Verbindung mit zweien der in $P, Q, O$ oder $O^{\prime}$ weitergehend, kann man schlieBlich den Raum kontinuierlich mit Weltlinienelementen überdecken, so daB durch die vorgegebenen Bestimmungsstücke der ganze Bewegungsverlauf völlig bestimmt ist. Man kommt so zu dem Schluß, daB im allgemeinen Falle die Freiheitsgrade der durch die Bornsche Bedingung gegebenen Bewegung genau die nämlichen sind, wie die der vierdimensionalen Bewegungsschraube, und da bei dieser die Bedingung erfüllt ist, so müssen die beiden Weltlinienbilder überhaupt zusammenfallen.

Eine Ausnahme tritt ein, wenn alle Elemente im Raume $l=0$ nicht nur auf den Strahlen vom Punkt $O$ aus, sondern auch auf dem Raum $l=0$ selbst senkrecht stehen, so daB alle ihre Normalräume in diesen Raum fallen. Dann kann die oben ausgeführte Konstruktion nicht zur Bestimmung von Richtungselementen auBerhalb dieses Raumes führen. In diesem Falle aber sind die Weltlinien die Orthogonaltrajektorien eines Systems linearer Räume, es handelt sich also um die auf p. 921 beschriebene Translation. Nach dem hier angedeuteten Beweis gilt auch für die räumliche Bewegung:

Die allgemeinsten Bewegungen, die der Differentialbedingung genügen können, sind die Translation und die gleichförmige Rotation.

\section{Schluß.}

Die Folgerungen, die aus unserem negativen Ergebnis bezüglich der Rotationen zu ziehen sind, sind wohl, daB die Bornsche Bedingung zu eng gestellt ist, um die Bewegung eines starren Körpers zu beschreiben. Sie versagt bei den zu fordernden Rotationen, wo sie nur auf den einfachen Fall der gleichförmigen Rotation führt, sie ist andererseits in ibrer differentiellen Form unnötig für die Translationen, die durch die endliche Formulierung auf p. 920 treffender und mehr im AnschluB an die Newtonsche Relativtheorie charakterisiert werden. Die Frage, die noch nicht beantwortet ist, ist die nach einer invarianten Definition, die für den Grenzfall $c=\infty$ in die alte übergeht. Und zwar einer Definition, die mindestens die Zahl der Freiheitsgrade hat, wie sie im Grenzfall $c=\infty$ auftreten, 
so daB sie bei endlichem $c$ und kleinen Geschwindigkeiten annähernd den starren Körper der alten Kinematik darstellt.

In Verallgemeinerung der Bornschen Translationen und der Bewegungen im Falle $c=\infty$ wäre es am naheliegendsten, als starren Körper ein dreidimensionales, ebenes System aufzufassen, das in einem beliebig veränderlichen Lorentzkoordinatensystem, in dessen $x^{\prime} y^{\prime} z^{\prime}$-Raum es enthalten ist, seine Gestalt nicht verändert, das, mit anderen Worten, die $\infty^{10}$ von einem Parameter abhängigen Bewegungen ausführen kann, bei denen alle Entfernungen $z$ wischen $z$ wei beliebigen Punkten:

$$
\left(x_{1}-x_{2}\right)^{2}+\left(y_{1}-y_{2}\right)^{2}+\left(z_{1}-z_{2}\right)^{2}-c^{2}\left(t_{1}-t_{2}\right)^{2}
$$

unverändert bleiben. Aus der so gewonnenen Parameterdarstellung der Bewegung kann der Parameter eliminiert werden, so daB seine Bedeutung unwesentlich ist und nur neun wesentliche Freiheitsgrade bleiben. Es scheint aber sehr fraglich, ob sich aus dieser Gruppe von $\infty^{9}$ Bewegungen durch geeignete Einschränkung eine invariant definierte Gruppe von $\infty^{6}$ Bewegungen aussondern läbt, die für $c=\infty$ in die $\infty^{6}$ starren Bewegungen der Newtonschen Kinematik übergingen und diesen dann genau entsprächen.

Es $^{1}$ ) ist ebenfalls unwahrscheinlich, daB sich durch irgend eine geeignet gewählte differentielle Formulierung der Starrheitsbedingungen gerade die Zahl der Freiheitsgrade wie in der alten Kinematik ergibt, die dort durch die endliche Form bestimmt werden, während die entsprechende Differentialbedingung zu einem überbestimmten System von Differentialgleichungen führt. Man wird aber in der erhöhten Zahl der Freiheitsgrade des starren Körpers kein Hindernis erblicken können, die zuletzt aufgestellte Definition doch als Ersatz für die starren Bewegungen der alten Kinematik zu betrachten. ${ }^{2}$ ) Wie in dieser kann nämlich die allgemeine Bewegung wieder durch Superposition von Translation und Rotation erzeugt werden. Der leichteren Anschaulichkeit halber beschränken wir uns hier auf den Fall von nur drei Koordinaten $x, y, t$. Zugrunde

1) Das Folgende ist bei der Korrektur anläBlich einer Anfrage von Hrn. Prof. W. Wien zugefügt.

2) Ich möchte nicht unerwähnt lassen, daB ich mehrfach Gelegenheit hatte, mit Hrn. Prof. Sommerfeld über diesen Punkt zu sprechen. 
legen wir die Bornschen Translationen, bei denen nach p. 921 der Übergang von einer Lage in die benachbarte des veränderlichen Koordinatensystems $x^{\prime} y^{\prime} t^{\prime}$ eine unendlich kleine Drehung (in dem dort definierten Sinne) um eine in der $x^{\prime} y^{\prime}$ Ebene selbst liegende Achse ist. Dieser Translation überlagern wir eine Rotation, d. h. eine Drehung der $x^{\prime} y^{\prime}$-Ebene in sich. Während nun aber in der alten Kinematik die Verschiebung des Drehpunktes dieser Rotation nur die Hinzufügung einer weiteren Translation bedeutet, kann jetzt nicht mehr die Rotation um einen beliebigen Punkt durch Rotation um einen anderen Punkt und eine Translation ersetzt werden, weil die Resultante aus zwei Rotationen keine Bornsche Translation geben kann. Daher führt die Superposition ron Translation und Rotation jetzt zu einer erhöhten Zahl der Freiheitsgrade. Wie aber die Bornschen Translationen, für die ja Unterlichtgeschwindigkeit vorauszusetzen ist, nur durch verfeinerte MeBmethoden von denen der alten Kinematik zu unterscheiden sind, da bei ihnen die $x^{\prime} y^{\prime}$-Ebenen immer in dem Raume zwischen der Ebene $t=0$ und dem Kegel $x^{2}+y^{2}-c^{2} t^{2}=0$ liegen, so sind auch die hinzukommenden drei Freiheitsgrade so beschränkt, daß sie praktisch nicht merkbar sind. Für $c=\infty$ endlich fallen alle neun Freiheitsgrade in die sechs der alten Kinematik zusammen.

Für die Dynamik wird es in ähnlicher Weise bei $\mathrm{Zu-}$ grundelegung dieser Kinematik und eines geeigneten dynamischen Prinzips unmöglich sein, das am starren Körper angreifende Kräftesystem wie im Grenzfall $c=\infty$ in eine Einzelkraft an einem "Schwerpunkt" und Momente um den Schwerpunkt zu zerlegen. Wenn aber ein solches dynamisches Prinzip es ermöglicht, die Bornsche Translation durch Einzelkräfte zu erzeugen, so werden auch durch Kraftsysteme die Rotationen und damit die $\infty^{\circ}$ Bewegungen des starren Körpers zu realisieren sein.

Daran knüpft sich noch eine Bemerkung über die Stellung des Michelson-Versuches (den man sich ja auch mit Bezug auf die Erdrotation ausgeführt denken kann), zu dem Nachweis, daB die von Born geforderten beschleunigten Rotationen nicht möglich sind. Die Volumelemente im Inneren der Erde verhielten sich, entsprechend der Bornschen Bedingung, wie 
944 F. Noether. Zur Kinematik des starren Körpers usw.

der Michelson-Apparat, wenn sie frei beweglich wären, denn nur für diesen Fall kann die Erfahrung am MichelsonApparat übertragen werden, und diese Tendenz muB in ihrem elastischen Verhalten zum Ausdruck kommen. Da sich eine solche Bewegung aber im Verband des starren Körpers kinematisch als unmöglich erwiesen hat, haben die inneren Reaktionen einen anderen Bewegungszustand zur Folge, wenn wir die Erde als starren Körper betrachten. Da die an der Oberfläche befindlichen freien Körper dagegen sich verhalten wie der Michelson-Apparat, so muB man schlieBen, daB theoretisch durch Vergleich ihres Verhaltens mit dem der Erdoberfläche sogar die Erdrotation nachzuweisen wäre. Natürlich ist darin kein Widerspruch gegen das Relativitätsprinzip $z u$ erblicken. München, Dezember 1909.

(Eingegangen 27. Dezember 1909). 